Torsten Wilholt

\title{
Soziale Erkenntnistheorie
}

(Manuskriptversion. Originalbeitrag erschienen in Information Philosophie 5/2007, 46-53.)

\section{Die individualistische Tradition der Erkenntnistheorie}

Wenn sich die Erkenntnistheorie spezifischen Quellen des Wissens zuwendet, dann geht es dabei traditionell fast immer um dreierlei: um sinnliche Wahrnehmung, um Erinnerung und um schlussfolgernde Verstandestätigkeit. Diese Quellen, die ohne Zweifel alle von elementarer Bedeutung für unser Wissen sind, lenken die Aufmerksamkeit ganz auf die kognitive Tätigkeit und Situation des Individuums. Die individualistische Ausrichtung der Erkenntnistheorie ist zumindest auf den zweiten Blick ein wenig merkwürdig: Jeder Mensch wird nach kurzer Überlegung feststellen, dass ein ganz überwiegender Teil seines Wissens das Ergebnis sozialer Vorgänge ist. Denn erstens stammt der Löwenanteil seines Wissens nicht (allein) aus eigener Anwendung der drei genannten individuellen epistemischen Vermögen, sondern beruht auf Mitteilung anderer. Und zweitens ergeben sich gerade heute viele wichtige Wissenserwerbsleistungen (in den Wissenschaften, im Journalismus oder im Rechtssystem) aus Gemeinschaftsunternehmungen vieler, deren Erfolge auch auf bestimmten sozialen Organisationsweisen beruhen. Sie fördern oft Wissensbestände zu Tage, die für uns alle von erheblicher Bedeutung sind und die für einzelne Individuen unerreichbar gewesen wären.

Dass aus diesen Gründen die individualistische Sichtweise der Erkenntnistheorie aufgegeben oder wenigstens um eine Erforschung der genuin sozialen Quellen unseres Wissens ergänzt werden müsste, ist in der Philosophie allerdings oft abgestritten worden. Schon David Hume behauptete in der Enquiry concerning Human Understanding (sec. X), dass bei Überzeugungen, die auf das Zeugnis anderer zurückgehen, unsere epistemische Rechtfertigung auf unseren individuellen Erfahrungen mit der Verlässlichkeit solchen Zeugnisses beruht. Dies wird üblicherweise als Argument dafür verstanden, dass soziale Quellen des Wissens nicht von eigener Art sind, sondern sich auf die drei genannten individuellen Quellen zurückführen lassen. Und was den sozialen Charakter beispielsweise des Gemeinschaftsunternehmens Naturwissenschaft angeht, so war sich die Mehrheit der Wissenschaftstheoretiker lange einig, ihr besonderer Erkenntniserfolg beruhe auf der Überlegenheit ihrer Methoden, deren Regeln prinzipiell auch von einem einzelnen Individuum erfolgreich angewandt 
werden könnten. Dass die schiere Menge der Forschungsarbeit eine arbeitsteilige Organisation des Unterfangens erforderlich mache, sei demnach eine bloß nachgeordnete und unwesentliche Erscheinung.

Solch individualistische Standpunkte sind zwar auch früher nicht ohne Ausnahmen geblieben. (Schon Thomas Reid wollte in seinen Essays on the Intellectual Powers of Man das Zeugnis anderer als zentrale, eigenständige Quelle von Rechtfertigung und Wissen verstanden wissen. Einflussreiche Ideen zum wesentlich gemeinschaftlichen Charakter von Wissen und Rechtfertigung finden sich auch etwa in Charles Sanders Peirces 'The Fixation of Belief' und in Ludwig Wittgensteins Über Gewißheit.) Doch eine eigene Dynamik entwickeln die Bemühungen, die sozialen Aspekte des Wissenserwerbs als eigenständiges philosophisches Forschungsthema zu würdigen, erst seit ungefähr drei Jahrzehnten. Unter dem Begriff social epistemology werden dabei oft sehr verschiedene Ansätze zusammengefasst. Ich werde im Folgenden zwei besonders einflussreiche Diskussionslinien vorstellen und dabei besonderes Augenmerk auf die Frage richten, aus welchen Gründen sich überhaupt das Erfordernis einer spezifisch sozialen Erkenntnistheorie ergibt.

\section{Wissen durch Mitteilung anderer}

Worin besteht bei Überzeugungen, zu denen wir auf der Grundlage von Mitteilung anderer gelangt sind - kurz: bei mitteilungsbasierten Überzeugungen unsere Rechtfertigung? Um diese Frage hat sich unter dem Schlagwort "testimony" eine lebendige Debatte entwickelt. In ihrem Kern steht die Kritik des auf Hume zurückgehenden sogenannten (testimonialen) Reduktionismus, worunter die Auffassung verstanden wird, in einer mitteilungsbasierten Überzeugung könne nur gerechtfertigt sein, wer auch von der Verlässlichkeit der fraglichen Mitteilung überzeugt sei und für diese Überzeugung wiederum Rechtfertigungsgründe besitze, die sich letztlich nicht auf Mitteilung anderer stützten. Die hauptsächliche Stoßrichtung der Kritik an dieser Position ist, dass es für fast alle Fälle mitteilungsbasierter Überzeugungen nicht realistisch sei, dass tatsächlich eine solche Rechtfertigung, die letztlich auf der Grundlage nicht-mitteilungsbasierten Wissens beruht, vorliege. Der Reduktionist rede somit ungewollt einem testimonialen Skeptizismus das Wort, demzufolge fast alle unsere mitteilungsbasierten Überzeugungen ungerechtfertigt seien.

Die Hoffnung, dass wir gerechtfertigte Überzeugungen über die Verlässlichkeit von Mitteilungen letztlich auf der Grundlage rein individueller Beobachtungen, die selbst mitteilungsunabhängig sind, ausgebildet haben könnten, hat insbesondere C.A.J. Coady (3, Kap. 9) durch Argumente unterminiert, 
die zeigen, dass die Menge der Beobachtungsüberzeugungen, die nicht ihrerseits zumindest teilweise durch mitteilungsbasiertes Wissen gerechtfertigt sind, sehr viel kleiner ist, als es zunächst den Anschein haben mag. Der durchschlagendste Faktor ist dabei, dass scheinbar reine Beobachtungsüberzeugungen oft bereits in ihren begrifflichen Grundlagen auf Hintergrundwissen beruhen, das seinerseits durch Mitteilung erworben wurde. Um etwa die Beobachtungsüberzeugung zu erlangen, dass dort ein Polizist auf der Kreuzung steht, muss ich über den Begriff des Polizisten verfügen; dies wiederum setzt voraus, dass ich einiges grundlegendes Wissen über die Institution der Polizei besitze. Gerade in der deshalb so wichtigen Phase, in der Kindern zugleich die Sprache und ein Großteil ihrer Alltagsüberzeugungen durch andere vermittelt wird, scheint es wenig plausibel, ihnen bereits durch Erfahrung ausreichend gerechtfertigte Überzeugungen über die Verlässlichkeit etwa der Mitteilungen ihrer Eltern zuzuschreiben.

Einige Erkenntnistheoretiker haben hieraus die Konsequenz gezogen, dass eine Position, die der Überzeugungsgewinnung durch Mitteilung anderer einen eigenen, grundlegenden und unabhängigen Status zubilligt, der menschlichen Wissenssituation besser gerecht wird als der Reduktionismus. So haben neben Coady auch etwa Tyler Burge und Richard Foley die Auffassung verteidigt, der bloße Umstand, dass jemandem von einem anderen eine Aussage als wahr präsentiert wird, liefere ihm auch einen prima facie guten Grund, sie zu akzeptieren. (Sie beleben damit einen bereits von Thomas Reid im Rahmen der CommonSense-Philosophie entwickelten Ansatz wieder.) Diese manchmal als defaultism und manchmal als credulism bezeichnete Grundposition macht Mitteilung durch andere zu einer eigenständigen, unabhängigen Rechtfertigungsquelle (nicht unähnlich den Sinneswahrnehmungen). Man kann sie durch ein Prinzip epistemischer Gleichheit motivieren, demzufolge es vernünftig ist, den Meinungen anderer prima facie keinen geringeren Status zuzubilligen als den eigenen.

Dem scheinbar naheliegenden Einwand, der Credulismus wolle Leichtgläubigkeit, Kritiklosigkeit und andere offensichtliche kognitive Defizite zum Erkenntnisprinzip erheben, können die Vertreter dieser Position mit einer Erläuterung begegnen: Der Status von Mitteilungen anderer als Rechtfertigungsquelle gilt nur so lange, wie der Empfänger oder die Empfängerin der Mitteilung keine dem entgegenstehenden Überzeugungen besitzt - weder solche, die den Inhalt der Mitteilung, noch die die Verlässlichkeit der mitteilenden Person in Frage stellen. In diesem Sinn ist zwar die Mitteilung durch andere als eigene Überzeugungs- und Rechtfertigungsquelle zu jedem Zeitpunkt durch das System unserer übrigen Überzeugungen eingeschränkt. Das soll aber wohlgemerkt 
nicht bedeuten, dass aus dieser Sicht bei der Bildung einer neuen, gerechtfertigten Überzeugung auf Grundlage von Mitteilung anderer unsere übrigen Überzeugungen regulär zu Überprüfungs- oder anderen Schlussfolgerungsvorgängen herbeigezogen würden. Sie müssen eher wie ein Sieb vorhanden sein, um gegebenenfalls zweifelhafte Mitteilungen abzufangen, ohne aber in den übrigen Fällen zu den Rechtfertigungsgrundlagen zu gehören.

Eigenständige soziale Wissensquellen in so starker Weise einzuführen und zu behaupten, die durch sie hervorgerufenen Überzeugungen könnten im Allgemeinen prima facie gerechtfertigt sein, ohne dass unabhängige Grundlagen zu ihrer Stützung vorlägen, ist ein tiefer Einschnitt, der sich nicht gänzlich in gutem Einklang mit Intuitionen über epistemische Rechtfertigung befindet. (Bin ich wirklich gerechtfertigt in meiner Überzeugung, dass es in Australien keine Würgeschlangen gibt, nur weil mir einmal eine wildfremde Person diese Information in ernsthafter Weise vorgetragen hat?) Da erste Prinzipien sich nicht beweisen lassen, gilt es, die motivierenden Überlegungen auf den Prüfstand zu stellen - sie entspringen im Fall der credulisitschen Positionen dem Wunsch, einen testimonialen Skeptizismus zu vermeiden. Hier stellt sich die Frage, ob nicht eine moderate und weniger kontraintuitive Zwischenposition dasselbe leisten könnte. So kann man etwa eingestehen, dass zwar eine globale Reduktion all unserer mitteilungsbasierter Überzeugungen auf mitteilungsunabhängige Rechtfertigungsquellen aussichtslos ist, dass man aber dennoch für viele mitteilungsbasierte Überzeugungen eine lokale Reduktion ihrer Rechtfertigung auf unabhängige Grundlagen (d.h. gute Gründe, von der Verlässlichkeit der Mitteilung ausgehen zu dürfen) erwarten darf. Insbesondere kann man postulieren, dass bei Erwachsenen solche unabhängigen Gründe für eine gerechtfertigte Übernahme von Überzeugungen anderer erforderlich sind, während in der kognitiven Entwicklungsphase eines Menschen blindes Vertrauen eine so unerlässliche Rolle spielt, dass hier ein auf diese Zeit begrenzter Credulismus unsere epistemische Rechtfertigung regiert. (Elizabeth Fricker hat schlagkräftig für die Möglichkeit solcher Zwischenpositionen argumentiert.) Ein genauer Konsens der sozialen Erkenntnistheorie über die überzeugendste Position im Spannungsfeld zwischen Reduktionismus und Credulismus ist zwar nicht auszumachen, wohl aber darüber, dass die Struktur unserer epistemischen Rechtfertigungsgründe in mehr als nur oberflächlicher Weise auf sozialen Grundlagen fußt. Der Mensch ist ein kognitives Gemeinschaftswesen, und sein epistemisches Vertrauen auf andere lässt sich jedenfalls nicht gänzlich auf das Wirken seiner übrigen kognitiven Vermögen reduzieren. 
Die gemeinschaftliche Wissensgewinnung in den Wissenschaften

Die Idealvorstellung, die Wissenschaft, das paradigmatische epistemische Gemeinschaftsunternehmen, schöpfe ihren Erfolg aus der Anwendung individueller, objektiver Methoden (also Methoden, die dieselben Ergebnisse zu zeitigen tendieren, gleich von welchem Individuum sie angewandt werden), hat spätestens seit den 1960er Jahren nachhaltig zu bröckeln begonnen. Thomas Kuhn und insbesondere spätere, durch ihn angeregte Wissenschaftshistoriker und -soziologen zeigten auf, dass der wissenschaftliche Konsens oft eher durch den Einfluss von Autorität zustande kommt als durch die Überzeugungskraft unbestechlicher Methoden; sie beschrieben die Wissenschaften als Arbeitsfeld, in dem das Handeln der einzelnen ebenso wie in anderen Lebensbereichen durch den Wunsch nach persönlichem Fortkommen angetrieben ist und weniger durch das unbedingte Streben nach der Wahrheit. Viele schlossen aus solchen Beobachtungen sogleich, eine Erkenntnistheorie der Wissenschaften im hergebrachten Sinn, die auf ihre Wahrheitsorientierung abziele und den vermeintlich rationalen und objektiven Charakter ihrer Wissensgewinnung zu erklären versuche, sei obsolet geworden.

Doch ist diese Schlussfolgerung wirklich so offensichtlich? Philip Kitcher hat überzeugend argumentiert, dass das Streben der Forscher nach in Karrierevorteile umsetzbarer wissenschaftlicher Anerkennung ein wichtiger Mechanismus ist, um eine effiziente kognitive Arbeitsteilung herzustellen (5, Kap. 8). Im Allgemeinen wird wissenschaftliche Anerkennung nach einer Prioritätsregel verteilt, der zufolge sie nur dem ersten Forscher, der ein bestimmtes Ergebnis liefert, zugesprochen wird. Die Aussichten darauf hängen deshalb für einen individuellen Forscher nicht nur vom intrinsischen Erfolgspotenzial des von ihm betriebenen Forschungsansatzes ab, sondern auch von der Gesamtzahl der gleichzeitig diesen Ansatz mit demselben Ziel verfolgenden Forscher. Ist diese Zahl zu groß, kann der individuelle Forscher wahrscheinlich seine Aussichten auf wissenschaftliche Anerkennung vergrößern, wenn er zu einem anderen, weniger überlaufenen Forschungsansatz wechselt - selbst wenn dieser ein geringeres intrinsisches Erfolgspotenzial hat. Auf diese Weise ist sicher gestellt, dass ein Teil des gesamten Forschungsaufwandes auch auf Vorhaben verwandt wird, die aus Sicht der wissenschaftlichen Orthodoxie weniger als optimale Erfolgsaussichten besitzen. Dass ein solcher Pluralismus der Ansätze für einen gemeinschaftlichen Erkenntnisfortschritt vorteilhaft ist, ist in der Philosophie schon seit langem vertreten worden (am Prominentesten von John Stuart Mill). Ähnlich wie Kitcher hat auch David Hull das individuelle Erfolgsstreben der Forscher als wichtiges zum gemeinschaftlichen Erkenntnisfortschritt beitragen- 
des Moment identifiziert. Aus seiner Sicht spielt es diese Rolle, weil es Forschern einen Anreiz gibt, fremde Forschungsergebnisse zu überprüfen: Die Überprüfung findet nicht fortwährend und selbstverständlich statt, aber sie wird vorgenommen, sobald ein Forscher den Verdacht schöpft, die Verlässlichkeit oder Fruchtbarkeit seiner eigenen Arbeit (und damit seine Aussicht auf Erfolg und Anerkennung) könnte durch einen möglichen Fehler im Ergebnis eines anderen bedroht sein.

Dass andererseits Wissenschaftler auch häufig auf den Ergebnissen anderer aufbauen, ohne diese selbst zu überprüfen, liegt Kitcher zufolge tatsächlich daran, dass sie sich in gewissem Sinn auf deren kognitive Autorität verlassen nämlich insofern sich diese Autorität auf die Akkumulation wissenschaftlicher Anerkennung und deren sichtbare Zeichen (Dienststellung, prestigeträchtige Publikationen, etc.) stützt. Indem diese kognitive Autorität die Entscheidung zur ungeprüften Übernahme eines Ergebnisses ermöglicht, werden Vorhaben erst machbar, die sonst am überbordenden Überprüfungsaufwand scheitern müssten. Dieser Vorteil überwiegt nach Kitcher selbst dann alle Nachteile, wenn die genannten Autoritätsmerkmale als Indikatoren für die Korrektheit der Ergebnisse nur wenig verlässlich sind.

Gemeinsam ist diesen Argumenten, dass Faktoren, die auf individueller Ebene betrachtet der Suche nach Wissen nicht zuträglich $\mathrm{zu}$ sein scheinen, durchaus einen positiven Beitrag zu einem sozial organisierten kognitiven Gemeinschaftsunternehmen leisten können. Prioritätsregel, Verknüpfung von Anerkennung und Karrierevorteilen, kognitive Autorität und andere soziale Arrangements verzerren also nicht etwa die wissensorientierte Methodik der Wissenschaften, sondern sie sind ein unverzichtbarer Teil von ihr. Aufbauend auf dieser Einsicht definiert Kitcher als allgemeines Problem der sozialen Erkenntnistheorie, die Eigenschaften epistemisch gut gestalteter sozialer Systeme zu identifizieren $(5$, S. 303).

\section{Einwände gegen das Bild der wertfreien Wissenschaft}

Zusätzlich zu den Beobachtungen der Historiker und Soziologen haben auch philosophische Erwägungen das Bild der objektiven wissenschaftlichen Methoden angekratzt. Besonders einflussreich war dabei die (unter anderem von Willard V.O. Quine beschriebene) Unterbestimmtheit der Theorie durch die Erfahrung. Da jede Gesamtheit empirischer Belege grundsätzlich mit verschiedenen Theorien vereinbar ist, müssen in die Entscheidung der Wissenschaftler für eine bestimmte Theorie immer zusätzliche, nicht auf den empirischen Belegen beruhende Präferenzen hineinspielen. Wissenschaftstheoretiker (darunter wie- 
derum Kuhn) haben vehement bestritten, dass diese Präferenzen subjektiv sein müssten - sie spiegelten vielmehr die epistemischen Werte der Wissenschaft wie Einfachheit, Fruchtbarkeit, Reichweite, Konsistenz mit hergebrachten Erkenntnissen usw. wieder. In diesem Sinn (so beispielsweise Ernan McMullin) könne die Wertfreiheit der Wissenschaften weiter behauptet werden - als Freiheit von nicht-epistemischen (d.h. sozialen, politischen, moralischen) Werten.

Doch mittlerweile hat eine ausgedehnte Debatte über Werte in der Wissenschaft gezeigt, dass die Unterscheidung zwischen epistemischen und nichtepistemischen Werten keineswegs unproblematisch ist. Es ist nicht erwiesen, dass etwa Einfachheit oder Fruchtbarkeit bei Theorien ein Indikator ihrer Wahrheit sind - die Wertschätzung dieser Merkmale ist also nicht verbindlich aufgrund der Wahrheitsorientierung der Wissenschaft. Feministische Wissenschaftstheoretikerinnen haben beispielsweise kritisiert, der weit verbreitete „epistemische Wert“ der externen Konsistenz (neuer Theorien mit althergebrachten) sei eher Ausdruck der Zufriedenheit der ihn vertretenden Person mit dem status quo denn ein verlässlicher Wahrheitsindikator. Und selbst wenn sich die Liste der epistemischen Werte eindeutig festlegen ließe, bliebe noch das Problem, dass jeder von ihnen vage und interpretationsbedürftig ist und häufig mit den jeweils anderen konfligiert. Ihre Anwendung erfordert daher Werturteile, bei denen es schlichtweg unrealistisch ist, den Einfluss nicht-epistemischer Werte auszuschließen.

Ein schlagkräftiges Argument gegen die Idealvorstellung von den wertfreien Methoden der Wissenschaft wurde bereits in den 1950er Jahren von Richard Rudner und anderen vorgetragen und neuerdings (vor allem durch Heather Douglas) erneut in die Diskussion gebracht: Es setzt an dem Umstand an, dass keine empirische Hypothese je vollständig durch die Daten bestätigt ist. Um sich zur Akzeptanz einer Hypothese zu entschließen, muss ein Wissenschaftler festlegen, welcher Grad empirischer Bestätigung dafür hinreichend ist. Statistik und induktive Logik können dies nicht vorgeben. Stattdessen, so bereits Rudner, müssen die Forscher ihre Wahl des Mindestgrades an Bestätigung von ihrer Einschätzung abhängig machen, wie schwerwiegend die Folgen einer irrtümlichen Akzeptanz bzw. einer fälschlichen Zurückweisung der Hypothese wären. In sofern beruht das Akzeptieren von Hypothesen immer auf (nichtepistemischen) Werturteilen.

Wie lässt sich noch von der Objektivität der Wissenschaft sprechen?

Wie lässt sich im Lichte der unvermeidbaren Wertbeladenheit noch von der Objektivität der Wissenschaften sprechen, und wenn es nur im Sinne eines 
wünschenswerten Ziels wäre? Viele sehen genau hier die Bedeutung der sozialen Erkenntnistheorie: Wissenschaftliche Objektivität hat ihren Ort nicht auf der Ebene der Einzelschritte des Forschungsprozesses, sondern auf der Ebene der sozialen Arrangements, in deren Rahmen wissenschaftliche Praxis stattfinden. Auf dieser Ebene können sich die individuell einseitigen Werteinflüsse ausgleichen und sogar produktiv stimulieren. Besonders einflussreich sind hier die Überlegungen Helen Longinos (8 und 9), die vier Bedingungen für das Zustandekommen der für Objektivität erforderlichen kritischen Interaktionen identifiziert (im Einzelnen sind dies: öffentlich anerkannte Foren der Kritik, Offenheit für und Eingehen auf Kritik, bestimmte, öffentlich anerkannte gemeinsame Standards sowie die Gleichheit der intellektuellen Autorität). Longino versteht die Bedingungen als Normen, die den Kern ihrer Position des critical contextual empiricism ausmachen. Ähnlich wie Longino meint auch Miriam Solomon (10), dass ein sozialer Pluralismus verschiedener Ansätze erkenntnistheoretisch produktiv ist, selbst wenn die Einzelpositionen einseitig wertbeladen sein mögen. (Allerdings versucht sie den Idealzustand der Wissenschaft nicht wie Longino prozedural, sondern konsequentialistisch als Ausgeglichenheit in der Summe aller Einseitigkeiten zu bestimmen.)

Auffällig ist an diesen Ideen ihr normativer Charakter: Mit dem verschwindenden Ideal der Wertfreiheit verflüchtigt sich auch die scharfe Abgrenzung zwischen der Erkenntnistheorie der Wissenschaften einerseits und wissenschaftspolitischen und -ethischen Überlegungen andererseits. Longinos Normen der Objektivität sind ein gutes Beispiel für einen Ansatz, der in untrennbarer Weise beide Bereiche zugleich betrifft. Für die Wissenschaftstheorie entstehen ganz deutlich neue Verknüpfungen mit sozialen und politischen Diskussionen, die, nebenbei gesagt, manchen ihrer Gründerväter aus den Reihen des Wiener Kreises (wie etwa Otto Neurath) gut gefallen hätten. Auch Kitcher hat sich dieser normativen Seite der sozialen Erkenntnistheorie zugewandt (6). Aus seiner Sicht äußert sich die unverzichtbare Wertbeladenheit vor allem in der Auswahl der Forschungsthemen - schließlich verfolgen die Wissenschaften nicht schlichtweg das Ziel, möglichst viele Wahrheiten gleich welcher Art zu finden, sondern sie konzentrieren sich auf die Suche nach bestimmten, signifikanten Wahrheiten. Genau dieser Begriff der Signifikanz ist aber nicht unabhängig von Wertentscheidungen zu bestimmen. Kitchers Ideal der well-ordered science zufolge sollte er sich nach demokratischen Prinzipien richten (wobei sich Kitcher an Idealen der deliberativen Demokratie zu orientieren versucht): Die Tagesordnung der Forschung sollte idealerweise diejenige sein, auf die ein Kollegium von wohlinformierten Repräsentantinnen und Repräsentanten sich nach einem fairen Beratschlagungsprozess einigen würde (6, Kap. 10). 
Für das Projekt einer eigenständigen sozialen Erkenntnistheorie gibt es also aus den Problemen von Erkenntnis- und Wissenschaftstheorie heraus mehrere verschiedene Motivationen. Erstens scheint die vollständige Zurückführung sozialer Quellen von Wissen und Rechtfertigung auf individuelle nicht zu funktionieren. Zweitens hat sich gezeigt, dass zu einem Verständnis der gemeinschaftlichen Wissensproduktion in den Wissenschaften auch eine Erklärung der sozialen Institutionen und Arrangements unverzichtbar ist, die ebenso zu ihrem Methodeninventar gehören wie kontrollierte Experimente oder statistische Datenauswertung. Drittens lässt sich das überkommene Idealbild der Wertfreiheit aller Forschung nicht aufrecht erhalten, was die Frage aufwirft, wie das Ziel wissenschaftlicher Objektivität neu zu deuten ist und wie gesellschaftliche Wertvorstellungen in produktiver und sinnvoller Weise in den wissenschaftlichen Prozess einfließen können.

Es gibt noch weitere, andersartige theoretische Motivationen, die ich aus Platzgründen unterschlagen habe. (So argumentiert beispielsweise Martin Kusch, epistemische Begriffe wie Wissen und Rechtfertigung seien selbst sozial konstituiert. Hintergrund dafür ist ihr normativer Charakter und die Auffassung, dass normative Phänomene nur in Gemeinschaften existieren könnten; vgl. 7.)

Daneben existieren aber auch viele bemerkenswerte Anwendungsfelder der sozialen Erkenntnistheorie (vgl. auch 4), die hier nur durch einige interessante Fragen angedeutet werden können: Wenn die sozialen Bedingungen der Wissensgewinnung entscheidend zum Erkenntnisprozess beitragen, wie wirken sich dann der wachsende Anwendungsdruck und die zunehmende Öknonomisierung der Wissenschaften erkenntnistheoretisch aus? Wie identifiziert man einen Experten, ohne selbst zum Experten werden zu müssen? Wie sollen wir angesichts der begrenzten Überzeugungskraft von Wertfreiheitsthesen mit wissenschaftlicher Expertise in der Politikberatung umgehen? Birgt es eine radikale Veränderung unserer Wissenssituation, dass wir mit Hilfe neuer Informationstechnologien auf soziale Quellen des Wissens mehr und mehr ebenso schnell und einfach zurückgreifen können wie auf unsere Erinnerung? Wie wirkt es sich auf die Massendistribution von Information durch kommerzielle Medien aus, wenn der Informationsempfänger nicht als Kunde fungiert, sondern als Ware (da das Geschäftsprinzip darin besteht, seine Aufmerksamkeit an Werbekunden zu verkaufen)? Neben den theoretischen Ausgangsfragen machen auch diese (und weitere) Anwendungsgebiete die soziale Erkenntnistheorie zu einem außerordentlich aktuellen Forschungsfeld. 


\section{Literatur zum Thema}

\section{Übersichtsdarstellungen:}

(1) Alvin Goldman: „What Is Social Epistemology? A Smorgasbord of Projects“, in: ders., Pathways to Knowledge: Private and Public, Oxford: Oxford University Press 2002, 182-204. Eine Variante dieser sehr reichhaltigen Überblicksdarstellung findet sich auch in der Stanford Encyclopedia of Philosophy, http://plato.stanford.edu/entries/

epistemology-social/

(2) Robert Audi: Epistemology: A Contemporary Introduction to the Theory of Knowledge, 2. Aufl. 2002, London, Routledge. In der neuen Auflage des bekannten Lehrbuches ist auch die soziale Erkenntnistheorie berücksichtigt (Kap. 5 u. 9).

\section{Weitere zentrale Literatur:}

(3) C.A.J. Coady: Testimony: A Philosophical Study, Oxford, Clarendon 1992.

(4) Alvin Goldman: Knowledge in a Social World, Oxford, Oxford University Press 1999.

(5) Philip Kitcher: The Advancement of Science, Oxford, Oxford University Press 1993.

(6) Philip Kitcher: Science, Truth and Democracy, Oxford, Oxford University Press 2001.

(7) Martin Kusch: Knowledge by Agreement: The Programme of Communitarian Epistemology, Oxford, Oxford University Press 2002.

(8) Helen E. Longino: Science as Social Knowledge: Values and Objectivity in Scientific Inquiry, Princeton, Princeton University Press 1990.

(9) Helen E. Longino: The Fate of Knowledge, Princeton, Princeton University Press 2003.

(10) Miriam Solomon: Social Empiricism, Cambridge, MA: MIT Press 2001.

Es existieren zwei eigene Fachzeitschriften zur sozialen Erkenntnistheorie: Social Epistemology, herausgegeben von Steve Fuller (seit 1987) und Episteme: A Journal of Social Epistemology, herausgegeben von Alvin Goldman (seit 2004). 